\title{
Neural Network Based Finite-Time Stabilization for Discrete-Time Markov Jump Nonlinear Systems with Time Delays
}

\author{
Fei Chen, ${ }^{1}$ Fei Liu, ${ }^{1}$ and Hamid Reza Karimi ${ }^{2}$ \\ ${ }^{1}$ Key Laboratory for Advanced Process Control of Light Industry of the Ministry of Education, School of Internet of Things Engineering, \\ Jiangnan University, Wuxi 214122, China \\ ${ }^{2}$ Department of Engineering, Faculty of Engineering and Science, University of Agder, 4898 Grimstad, Norway
}

Correspondence should be addressed to Fei Liu; fliu@jiangnan.edu.cn

Received 10 July 2013; Accepted 5 September 2013

Academic Editor: Lixian Zhang

Copyright (c) 2013 Fei Chen et al. This is an open access article distributed under the Creative Commons Attribution License, which permits unrestricted use, distribution, and reproduction in any medium, provided the original work is properly cited.

\begin{abstract}
This paper deals with the finite-time stabilization problem for discrete-time Markov jump nonlinear systems with time delays and norm-bounded exogenous disturbance. The nonlinearities in different jump modes are parameterized by neural networks. Subsequently, a linear difference inclusion state space representation for a class of neural networks is established. Based on this, sufficient conditions are derived in terms of linear matrix inequalities to guarantee stochastic finite-time boundedness and stochastic finite-time stabilization of the closed-loop system. A numerical example is illustrated to verify the efficiency of the proposed technique.
\end{abstract}

\section{Introduction}

Markov jump systems (MJSs) are an important class of stochastic dynamic systems, which are popular when modeling an abrupt change in the system structure and parameters, such as component failures or repairs, changing subsystem interconnections and environmental disturbance. This family of systems has great practical potential in a variety of fields, such as solar thermal central receivers systems, economic systems, communication systems, manufacturing systems, and networked control systems [1-4]. MJSs have been extensively studied since the pioneering work on quadratic control of MJSs [5], and many achievements have been made on Lyapunov stochastic stability and stabilization in the last three decades [6-18].

However, it is worth noting that the Lyapunov stochastically stable systems may not possess good or expected transient characteristics over a finite-time horizon. In many practical problems, it is of interest to investigate the stability of a system over a finite interval of time. For example, referring to aircraft control, it requests that, during the execution of a certain task, the state variables should not exceed some threshold under all admissible pilot inputs and in the presence of wind disturbances. Classical control theory does not directly address this requirement, because it focuses mainly on the asymptotic behavior of the system (over an infinite-time interval) and does not usually specify bounds on the trajectories. Therefore, it is necessary to limit the state in an acceptable region and consider finite-time stability (FTS) given by Dorato [19].

The concept of FTS has been further extended into finite-time boundness (FTB) [20, 21], when system possesses bounded exogenous disturbance. A linear matrix inequality (LMI) framework has been established to distinguish FTS and Lyapunov asymptotical stability [22-24]. Compared with Lyapunov stochastically stable condition, FTS relaxes the condition by allowing that the Lyapunov-like function can increase at every sampling time instant. That is why FTS is so attractive and widely used in practical engineering.

As MJSs are considered, a number of results on stochastic FTS or stochastic FTB have been developed [25-28], and recently, the obtained results have been extended to 
continuous-time MJSs with nonlinearities via fuzzy or neural network approach $[24,29,30]$. In order to make the stochastic systems more manageable and satisfy the requirements for finite-time behavior of a system in engineering fields, it motivates us to investigate the finite-time stability and stabilization problems for a class of MJSs. Furthermore, time delay is a common phenomenon and is inevitable in practice systems [31-33]. Due to the interaction among system dynamics, stochastic jumps, and time delays, the dynamics of MJSs with time delay become more complex than MJSs without time delay and time delay systems without jumps. So far, in comparison with the literatures available for continuous-time nonlinear MJSs with time delays, the corresponding FTS or FTB results for discrete-time nonlinear systems have been relatively few.

It is, therefore, the main purpose of this paper to shorten such a gap by investigating the finite-time stabilization problem for discrete-time nonlinear MJSs with time delays. With neural networks, the nonlinearities of MJSs are approximated firstly by linear difference inclusion under state-space representation. Then, a mode-dependent finite-time controller is developed to make the nonlinear MJSs stochastic finitetime stabilizable for all admissible approximation errors of the neural networks and the norm-bounded external disturbances. The controller gains could be derived by solving a set of LMIs. An attractive feature of the proposed scheme is that the coupling relationship between time delay and given finite-time horizon is explored by obtaining delayindependent conditions.

Notations in this paper are fairly standard. $R^{n}$ and $R^{n \times m}$ denote $n$-dimensional Euclidean space and the set of all the $n \times m$ real matrices, respectively; $A^{T}$ (or $x^{T}$ ) and $A^{-1}$ denote the transpose of the matrix $A$ (or the vector $x$ ) and the inverse of the matrix $A$, respectively. $\lambda_{\max }(A)$ and $\lambda_{\min }(A)$ denote, respectively, the maximal and minimal eigenvalues of a real matrix $A,\|A\|$ denotes the Euclidean norm of matrix $A, E\{\cdot\}$ denotes the mathematics statistical expectation of the stochastic process or vector, $l_{2}\left[\begin{array}{ll}0 & N\end{array}\right)$ is the space of summable infinite sequence over $\left[\begin{array}{ll}0 & N\end{array}\right), P>0$ stands for a positive-definite matrix, $I$ is the unit matrix with appropriate dimensions, and “*” means the symmetric terms in a symmetric matrix.

\section{System Description and Problem Formulation}

We consider a nonlinear discrete-time MJS, which can be described by the following mathematical model:

$$
\begin{gathered}
x_{k+1}=A\left(r_{k}\right) x_{k}+A_{d}\left(r_{k}\right) x_{k-d}+B\left(r_{k}\right) u_{k} \\
+B_{w}\left(r_{k}\right) w_{k}+C\left(r_{k}\right) f\left(x_{k}, r_{k}\right), \\
x_{f}=\varphi_{f}, \quad f \in\{-d, \ldots, 0\}, \quad r(0)=r_{0},
\end{gathered}
$$

where $x_{k} \in R^{n}$ is the vector of state variables, $u_{k} \in R^{m}$ is the controlled input, $f(\cdot)$ is a discrete nonlinear mapping with $f(0)=0$ but not assumed to be known a prior, and $w_{k} \epsilon$ $l_{2}^{q}\left[\begin{array}{ll}0 & +\infty\end{array}\right)$ is the exogenous disturbances satisfying

$$
\|w\|_{2}^{2}=E\left[\sum_{k=0}^{N} w_{k}^{T} w_{k}\right]<\delta^{2} .
$$

For each possible value of $r_{k}=i$, we denote

$$
\begin{gathered}
A\left(r_{k}\right)=A_{i}, \quad A_{d}\left(r_{k}\right)=A_{d i}, \quad B\left(r_{k}\right)=B_{i}, \\
B_{w}\left(r_{k}\right)=B_{w i}, \quad C\left(r_{k}\right)=C_{i}, \quad f\left(x_{k}, r_{k}\right)=f_{i}\left(x_{k}\right),
\end{gathered}
$$

where $r_{k}$ is a discrete-state Markov chain taking values in $M=$ $\{1,2, \ldots, s\}$ with transition probabilities

$$
\operatorname{Prob}\left\{r_{k+1}=j \mid r_{k}=i\right\}=\pi_{i j},
$$

where $\pi_{i j}$ is the transition probabilities from mode $i$ to mode $j$ that satisfies

$$
\pi_{i j} \geq 0, \quad \sum_{j=1}^{m} \pi_{i j}=1, \quad \forall i, j \in M
$$

For each mode $i$, nonlinear function $f_{i}\left(x_{k}\right)$ is to be parameterized by neural networks. Such parameterization makes sense because any nonlinear function can be approximated arbitrarily well on a compact interval by a neural network. Let the $L$-layered perceptrons $N_{i}\left(x_{k}, W_{r 1}, W_{r 2}, \ldots, W_{r L}\right)$ be suitably trained to approximate the nonlinear term $f_{i}\left(x_{k}\right)$, which is described in matrix-vector notation as

$$
\begin{aligned}
& N_{i}\left(x_{k}, W_{i 1}, W_{i 2}, \ldots, W_{i L}\right) \\
& \quad=\psi_{i L}\left[W_{i L} \cdots \psi_{i 2}\left[W_{i 2}\left[\psi_{i 1}\left[W_{i 1} x(t)\right]\right]\right]\right],
\end{aligned}
$$

where all the weight matrices $W_{i r} \in R^{n_{i r} \times n_{i(r-1)}}, r=1, \ldots$, $L$, from the $(r-1)$ th layer to the $(r-L)$ th layer will be determined via back propagation (BP) procedure [24]; the activation function vector of $r$ th layer is defined as $\psi_{i r}[\zeta]=\left[\phi_{i 1}\left(\varsigma_{i 1}\right), \phi_{i 2}\left(\varsigma_{i 2}\right), \ldots, \phi_{i n_{r}}\left(\varsigma_{i n_{r}}\right)\right]^{T}$, where $n_{r}$ indicates the neurons of $r$ th layer and let

$$
\phi_{i l}\left(\varsigma_{i l}\right)=\lambda_{i l}\left(\frac{1-e^{-\varsigma_{i l} / q_{i l}}}{1+e^{-\varsigma_{i l} / q_{i l}}}\right), \quad q_{i l}, \lambda_{i l}>0, l=1,2, \ldots, n_{r} .
$$

The maximum and minimum derivatives of activation function $\phi_{i l}$ are defined as follows:

$$
s_{i l}\left(m, \phi_{i l}\right)= \begin{cases}\min _{\zeta_{i l}} \frac{\partial \phi_{i l}\left(\zeta_{i l}\right)}{\partial \zeta_{i l}}, & m=0, \\ \max _{\zeta_{i l}} \frac{\partial \phi_{i l}\left(\zeta_{i l}\right)}{\partial \zeta_{i l}}, & m=1 .\end{cases}
$$

For $r$ th layer of neural network, activation function $\phi_{i l}$ can be rewritten as the following min-max form:

$$
\phi_{i l}=h_{i l}(0) s_{i l}\left(0, \phi_{i l}\right)+h_{i l}(1) s_{i l}\left(1, \phi_{i l}\right) \text {, }
$$


where $h_{i l}(m), m=0,1$, are a set of positive real numbers associated with $\phi_{i l}$ satisfying $h_{i l}(m)>0$ and $h_{i l}(0)+h_{i l}(1)=1$.

According to the approximation theorem, for given accuracy $\rho_{i}>0$, there exist ideal constant weight matrices $W_{i r}^{*}$ defined as

$$
\begin{aligned}
& \left(W_{i 1}^{*}, W_{i 2}^{*}, \ldots, W_{i L}^{*}\right) \\
= & \underset{\left(W_{i 1}^{*}, W_{i 2}^{*}, \ldots, W_{i L}^{*}\right)}{\arg \min }\left\{\max _{x_{k} \in D}\left\|f_{i}\left(x_{k}\right)-N_{i}\left(x_{k}, W_{i 1}, W_{i 2}, \ldots, W_{i L}\right)\right\|\right\},
\end{aligned}
$$

where $D$ is a compact set $D \in R^{m}$, such that

$$
\max _{x_{k} \in D}\left\|f_{i}\left(x_{k}\right)-N_{i}\left(x_{k}, W_{i 1}^{*}, W_{i 2}^{*}, \ldots, W_{i L}^{*}\right)\right\| \leq \rho_{i}\left\|x_{k}\right\| .
$$

For each mode $i$, denote a set of $n_{r}$ dimensional index vectors of the $r$ th layer as

$$
\gamma_{n_{r}}=\gamma_{n_{r}}\left(\sigma_{i}\right)=\left\{\sigma_{i} \in R^{n_{r}} \mid \sigma_{i l} \in\{0,1\}, l=1, \ldots, n_{r}\right\},
$$

where $\sigma_{i}$ is used as a binary indicator. Obviously, the $r$ th layer with $n_{r}$ neurons has $2^{n_{r}}$ combinations of binary indicator with $m=0,1$, and the elements of index vectors for all $L$ layers neural network have $2^{n_{L}} \times \cdots \times 2^{n_{2}} \times 2^{n_{1}}$ combinations in the set

$$
\Theta=\gamma_{n_{L}} \oplus \cdots \oplus \gamma_{n_{2}} \oplus \gamma_{n_{1}} .
$$

By using (8) and adopting the compact representation [34], the multilayer neural network (6) can be expressed as follows:

$$
\begin{aligned}
N\left(x_{x}, W_{i 1}^{*}, W_{i 2}^{*}, \ldots, W_{i L}^{*}\right) & =\psi_{i L}\left[W_{i L}^{*} \cdots \psi_{i 2}\left[W_{i 2}^{*}\left[\begin{array}{c}
\sum_{m=0}^{1} h_{i 11}(k) s_{i 11}\left(m, \phi_{i 11}\right) \times\left(W_{i 1}^{*} x\right)_{i 1} \\
\vdots \\
\sum_{m=0}^{1} h_{i 1 n_{1}}(k) s_{i 1 n_{1}}\left(m, \phi_{i 1 n_{1}}\right) \times\left(W_{i 1}^{*} x\right)_{i n_{1}}
\end{array}\right]\right] \cdots\right] \\
& =\sum_{\sigma_{i} \in \Theta} \mu_{\sigma_{i}} A_{\sigma_{i}}\left(\sigma_{i}, \psi_{i}, W_{i}^{*}\right) x_{k}
\end{aligned}
$$

where

$$
\begin{aligned}
A_{\sigma_{i}}= & \operatorname{diag}\left[s_{L i l}\left(\sigma_{L i l}, \phi_{L i l}\right)\right] W_{L}^{*} \cdots \operatorname{diag}\left[s_{2 i l}\left(\sigma_{2 i l}, \phi_{2 i l}\right)\right] \\
& \times W_{2}^{*} \operatorname{diag}\left[s_{1 i l}\left(\sigma_{1 i l}, \phi_{1 i l}\right)\right] W_{1}^{*}, \\
\sum_{\sigma_{i} \in \Theta} \mu_{\sigma_{i}}= & \sum_{m_{i L n_{L}}=0}^{1} \cdots \sum_{m_{i 1 n_{1}}=0}^{1} h_{i L n_{L}}\left(m_{i L n_{L}}\right) \cdots h_{i L 1}\left(m_{i L 1}\right) \\
\vdots & \vdots \\
m_{i L 1}=0 & m_{i 11}=0 \\
& \cdots h_{i 1 n_{1}}\left(m_{i 1 n_{1}}\right) \cdots h_{i 11}\left(m_{r 11}\right)=1 .
\end{aligned}
$$

Thus by means of multilayer neural network, the nonlinear MJS (1) is translated into a group of LDIs with error bounds, in which the different inclusion is powered by stochastic Markov process; that is,

$$
\begin{gathered}
x_{k+1}=\left[\sum_{\sigma_{i} \in \Theta} \mu_{\sigma_{i}} A_{\sigma_{i}}+A_{i}\right] x_{k}+A_{d i} x_{k-d} \\
+B_{i} u_{k}+B_{w i} w_{k}+C_{i} \Delta f_{i}\left(x_{k}\right), \\
x_{f}=\varphi_{f}, \quad f \in\{-d, \ldots, 0\}, \quad r(0)=r_{0},
\end{gathered}
$$

where

$$
\begin{aligned}
\Delta f_{i}\left(x_{k}\right) & =\max _{x_{k} \in D}\left\|f_{i}\left(x_{k}\right)-N_{i}\left(x_{k}, W_{i 1}^{*}, W_{i 2}^{*}, \ldots, W_{i L}^{*}\right)\right\| \\
& \leq \rho_{i}\left\|x_{k}\right\|
\end{aligned}
$$

denotes the approximation errors of networks.

Remark 1. The detailed structure and quantitative size of error dynamics $\Delta f_{i}\left(x_{k}\right)$ are not needed, but only normbounded assumption is required. This condition is easily satisfied in practical cases, such as bioinformatics system, medical diagnosis, fault diagnosis, and image and pattern recognition. Actually, the approximation error between the target function and the closest neural network function of a given network family can be made as small as desired by increasing the number of nodes [35]. Also the bounds of norm may vary according to different nonlinearities in different modes.

\section{Main Results}

Based on the LDI model (16) of networks, we consider the following discrete-time state feedback control law for nonlinear stochastic MJS (1):

$$
u_{k}=K_{i} x_{k} .
$$


The resulting closed-loop system can be obtained as follows:

$$
\begin{gathered}
x_{k+1}=\bar{A}_{i} x_{k}+A_{d}\left(r_{k}\right) x_{k-d}+B_{w}\left(r_{k}\right) w_{k}+\Delta f_{i}\left(x_{k}\right), \\
x_{f}=\varphi_{f}, \quad f \in\{-d, \ldots, 0\}, \quad r(0)=r_{0},
\end{gathered}
$$

where

$$
\bar{A}_{i}=\sum_{\sigma_{i} \in \Theta} \mu_{\sigma_{i}} A_{\sigma_{i}}+A_{i}+B_{i} K_{i}
$$

The aim of this paper is to find some sufficient conditions which guarantee stochastic finite-time boundness and stochastic finite-time stabilization of the closed-loop system (19). The general idea of finite-time control can be formalized through the following definitions over a finite-time interval for some given initial conditions.

Definition 2 (stochastic finite-time stability). A discrete-time nonlinear MJS (1) (setting $u_{k}=0$ and $w_{k}=0$ ) is said to be, stochastic finite-time stability (FTS) with respect to given $\left(c_{1}, c_{2}, G, N\right)$, where $c_{2}>c_{1}$ and $G>0$, if $E\left\{x_{k}^{T} G x_{k}\right\}<c_{2}^{2}$, $k \in\{1,2, \ldots, N\}$ whenever $\max _{k_{0}-d \leq k \leq k_{0}} E\left\{x_{0}^{T} G x_{0}\right\} \leq c_{1}^{2}$.

Definition 3 (stochastic finite-time boundness). A discretetime nonlinear MJS (1) (setting $u_{k}=0$ ) is said to be of stochastic finite-time boundness (FTB) with respect to $\left(c_{1}, c_{2}, G, N, \delta\right)$ with $c_{2}>c_{1}$ and $G>0$, if $E\left\{x_{k}^{T} G x_{k}\right\}<c_{2}^{2}, k \in$ $\{1,2, \ldots, N\}$, whenever $\max _{k_{0}-d \leq k \leq k_{0}} E\left\{x_{0}^{T} G x_{0}\right\} \leq c_{1}^{2}$.

Before proceeding further, we introduce the following lemmas which will be needed for the derivation of our main results.

Lemma 4. The closed-loop system (19) is stochastic FTB with respect to the given $\left(c_{1}, c_{2}, G, N, \delta\right)$ and scalar $\alpha \geq 0$, if there exist mode-dependent symmetric positive-definite matrix $P_{i}$ and symmetric positive-definite matrices $Q$ and $S$ such that

$$
\left[\begin{array}{cccc}
\bar{A}_{i}^{T} \bar{P}_{j} \bar{A}_{i}-(1+\alpha) P_{i}+Q & \bar{A}_{i}^{T} \bar{P}_{j} A_{d i} & \bar{A}_{i}^{T} \bar{P}_{j} B_{w i} & \bar{A}_{i}^{T} \bar{P}_{j} C_{i} \\
* & A_{d i}^{T} \bar{P}_{j} A_{d i}-Q & A_{d i}^{T} \bar{P}_{j} B_{w i} & A_{d i}^{T} \bar{P}_{j} C_{i} \\
* & * & B_{w i}^{T} \bar{P}_{j} B_{w i}-(1+\alpha) S & B_{w i}^{T} \bar{P}_{j} C_{i} \\
* & * & * & C_{i}^{T} \bar{P}_{j} C_{i}-(1+\alpha) R
\end{array}\right]
$$

$<0$,

$$
\begin{aligned}
c_{1}^{2} \max _{i \in M}\{ & \left.\lambda_{\max }\left(\widetilde{P}_{i}\right)\right\}+c_{1}^{2} d \lambda_{\max }(\widetilde{Q})+\delta^{2} \lambda_{\max }(S) \\
& +c_{2}^{2} \rho_{i}^{2} \lambda_{\max }(\widetilde{R})<\frac{c_{2}^{2} \min _{i \in M}\left\{\lambda_{\min }\left(\widetilde{P}_{i}\right)\right\}}{(1+\alpha)^{N}}
\end{aligned}
$$

where $\widetilde{P}_{i}=G^{-1 / 2} P_{i} G^{-1 / 2}, \widetilde{Q}=G^{-1 / 2} Q G^{-1 / 2}, \widetilde{R}=$ $G^{-1 / 2} R G^{-1 / 2}$, and $\lambda_{\max }(\cdot), \lambda_{\min }(\cdot)$ indicate the maximal and minimal eigenvalues of the augment, respectively.
Proof. For the closed-loop system (19), choose a stochastic Lyapunov function candidate as

$$
V_{i}(k)=x_{k}^{T} P_{i} x_{k}+\sum_{f=k-d}^{k-1} x_{f}^{T} Q x_{f}
$$

Simple calculation shows that

$$
\begin{aligned}
E\left\{V_{i}\right. & (k+1)\}-V_{i}(k) \\
= & x_{k}^{T}\left(\bar{A}_{i}^{T} \bar{P}_{j} \bar{A}_{i}-P_{i}+Q\right) x_{k}+2 x_{k}^{T} \bar{A}_{i}^{T} \bar{P}_{j} A_{d i} x_{k-d} \\
& +2 x_{k}^{T} \bar{A}_{i}^{T} \bar{P}_{j} B_{w i} w_{k}+2 x_{k}^{T} \bar{A}_{i}^{T} \bar{P}_{j} C_{i} \Delta f_{i}\left(x_{k}\right) \\
& +x_{k-d}^{T}\left(A_{d i}^{T} \bar{P}_{j} A_{d i}-Q\right) x_{k-d} \\
& +2 x_{k-d}^{T} A_{d i}^{T} \bar{P}_{j} B_{w i} w_{k}+2 x_{k-d}^{T} A_{d i}^{T} \bar{P}_{j} C_{i} \Delta f_{i}\left(x_{k}\right) \\
& +w_{k}^{T} B_{w i}^{T} \bar{P}_{j} B_{w i} w_{k} \\
& +2 w_{k}^{T} B_{w i}^{T} \bar{P}_{j} C_{i} \Delta f_{i}\left(x_{k}\right)+\Delta f_{i}^{T}\left(x_{k}\right) C_{i}^{T} \bar{P}_{j} C_{i} \Delta f_{i}\left(x_{k}\right) \\
= & \zeta_{k}^{T} \Omega_{i} \zeta_{k},
\end{aligned}
$$

where

$$
\begin{gathered}
\bar{P}_{j} \triangleq \sum_{j=1}^{s} \pi_{i j} P_{j}, \quad \zeta_{k}=\left[\begin{array}{llll}
x_{k}^{T} & x_{k-d}^{T} & w_{k}^{T} & \Delta f_{i}^{T}\left(x_{k}\right)
\end{array}\right]^{T}, \\
\Omega_{i}=\left[\begin{array}{cccc}
\bar{A}_{i}^{T} \bar{P}_{j} \bar{A}_{i}-P_{i}+Q & * & * & * \\
A_{d i}^{T} \bar{P}_{j} \bar{A}_{i} & A_{d i}^{T} \bar{P}_{j} A_{d i}-Q & * & * \\
B_{w i}^{T} \bar{P}_{j} \bar{A}_{i} & B_{w i}^{T} \bar{P}_{j} A_{d i} & B_{w i}^{T} \bar{P}_{j} B_{w i} & * \\
C_{i}^{T} \bar{P}_{j} \bar{A}_{i} & C_{i}^{T} \bar{P}_{j} A_{d i} & C_{i}^{T} \bar{P}_{j} B_{w i} & C_{i}^{T} \bar{P}_{j} C_{i}
\end{array}\right] .
\end{gathered}
$$

Conditions (21) and (24) imply that

$$
\begin{aligned}
E\left\{V_{i}(\right. & +1)\} \\
\leq & (1+\alpha) x_{k}^{T} P_{i} x_{k}+(1+\alpha) w_{k}^{T} S w_{k} \\
& +(1+\alpha) \Delta f_{i}^{T}\left(x_{k}\right) R \Delta f_{i}\left(x_{k}\right)+(1+\alpha) \sum_{f=k-d}^{k-1} x_{f}^{T} Q x_{f} \\
= & (1+\alpha) V_{i}(k)+(1+\alpha) w_{k}^{T} S w_{k} \\
& +(1+\alpha) \Delta f_{i}^{T}\left(x_{k}\right) R \Delta f_{i}\left(x_{k}\right) .
\end{aligned}
$$

Noting that $\alpha \geq 0$, we can obtain from (26) that

$$
\begin{aligned}
V_{i}(k) \leq & (1+\alpha)^{k} V_{i}(0)+\sum_{f=1}^{k}(1+\alpha)^{k-f+1} w_{f-1}^{T} S w_{f-1} \\
& +\sum_{f=1}^{k}(1+\alpha)^{k-f+1} c_{2}^{2} \rho_{i}^{2} \lambda_{\max }(\widetilde{R})
\end{aligned}
$$




$$
\begin{aligned}
=(1+\alpha)^{k}[ & x_{0}^{T} P_{i} x_{0}+\sum_{f=-d}^{-1} x_{f}^{T} Q x_{f} \\
& +\sum_{f=1}^{k}(1+\alpha)^{1-f} w_{f-1}^{T} S w_{f-1} \\
& \left.+\sum_{f=1}^{k}(1+\alpha)^{1-f} c_{2}^{2} \rho_{i}^{2} \lambda_{\max }(\widetilde{R})\right] \\
\leq(1+\alpha)^{N}[ & c_{1}^{2} \max _{i \in M}\left\{\lambda_{\max }\left(\widetilde{P}_{i}\right)\right\}+c_{1}^{2} d \lambda_{\max }(\widetilde{Q}) \\
& \left.+\delta^{2} \lambda_{\max }(S)+c_{2}^{2} \rho_{i}^{2} \lambda_{\max }(\widetilde{R})\right] .
\end{aligned}
$$

Note that

$$
\begin{aligned}
V_{i}(k) & =x_{k}^{T} P_{i} x_{k}+\sum_{f=k-d}^{k-1} x_{f}^{T} Q x_{f} \\
& \geq x_{k}^{T} P_{i} x_{k} \\
& \geq \min _{i \in M}\left\{\lambda_{\min }\left(\widetilde{P}_{i}\right)\right\} x_{k}^{T} G x_{k} .
\end{aligned}
$$

According to (27)-(28), one has

$$
\begin{aligned}
x_{k}^{T} G x_{k} \leq & \left(( 1 + \alpha ) ^ { N } \left(c_{1}^{2} \max _{i \in M}\left\{\lambda_{\max }\left(\widetilde{P}_{i}\right)\right\}+c_{1}^{2} d \lambda_{\max }(\widetilde{Q})\right.\right. \\
& \left.\left.+\delta^{2} \lambda_{\max }(S)+c_{2}^{2} \rho_{i}^{2} \lambda_{\max }(\widetilde{R})\right)\right) \\
& \times\left(\min _{i \in M}\left\{\lambda_{\min }\left(\widetilde{P}_{i}\right)\right\}\right)^{-1}
\end{aligned}
$$

Condition (19) implies that, for $k \in\{1,2, \ldots, N\}, E\left\{x_{k}^{T} G x_{k}\right\}<$ $c_{2}^{2}$. This completes the proof.

Now, we direct our attention to present a solution to the problem of finite-time stabilizing controller design. Such controller is provided by the following theorem.

Theorem 5. The closed-loop system (19) is stochastic finitetime stabilizable via state feedback with respect to the given $\left(c_{1}, c_{2}, G, N, \delta\right)$ and scalar $\alpha \geq 0$, if there exist matrices $X_{i}=$ $X_{i}^{T}>0, Y_{i}, H=H^{T}>0, S=S^{T}>0$, and $R=R^{T}>0$ and scalars $\lambda_{1}, \lambda_{2}, \lambda_{3}, \lambda_{4}>0$ such that

$$
\left[\begin{array}{ccccc}
-(1+\alpha) X_{i} & N_{1 i}^{T} & 0 & 0 & X_{i} \\
N_{1 i} & -M_{5 i}+N_{5 i} & M_{3 i} & M_{4 i} & 0 \\
0 & M_{3 i}^{T} & -(1+\alpha) S & 0 & 0 \\
0 & M_{4 i}^{T} & 0 & -(1+\alpha) R & 0 \\
X_{i} & 0 & 0 & 0 & -H
\end{array}\right]
$$

$$
\begin{gathered}
\lambda_{1} G^{-1}<X_{i}<G^{-1}, \quad \lambda_{2} G^{-1}<H, \quad S<\lambda_{3} I, \quad R<\lambda_{4} G \\
{\left[\begin{array}{ccc}
-\frac{c_{2}^{2}}{(1+\alpha)^{N}}+\delta^{2} \lambda_{3}+c_{2}^{2} \rho_{i}^{2} \lambda_{4} & c_{1} & \sqrt{d} c_{1} \\
c_{1} & -\lambda_{1} & 0 \\
\sqrt{d} c_{1} & 0 & -\lambda_{2}
\end{array}\right]<0}
\end{gathered}
$$

Proof. By using Schur complement, from condition (21) in Lemma 4, it follows that

$$
\left[\begin{array}{ccccc}
-(1+\alpha) P_{i}+Q & * & * & * & * \\
0 & -Q & * & * & * \\
0 & 0 & -(1+\alpha) S & * & * \\
0 & 0 & 0 & -(1+\alpha) R & * \\
M_{1 i} & M_{2 i} & M_{3 i} & M_{4 i} & -M_{5 i}
\end{array}\right] \leq 0
$$

where

$$
\begin{aligned}
M_{1 i} & =\left[\sqrt{\pi_{i 1}} \bar{A}_{i}^{T}, \ldots, \sqrt{\pi_{i s}} \bar{A}_{i}^{T}\right]^{T}, \\
M_{2 i} & =\left[\sqrt{\pi_{i 1}} A_{d i}^{T}, \ldots, \sqrt{\pi_{i s}} A_{d i}^{T}\right]^{T}, \\
M_{3 i} & =\left[\sqrt{\pi_{i 1}} B_{w i}^{T}, \ldots, \sqrt{\pi_{i s}} B_{w i}^{T}\right]^{T}, \\
M_{4 i} & =\left[\sqrt{\pi_{i 1}} C_{i}^{T}, \ldots, \sqrt{\pi_{i s}} C_{i}^{T}\right]^{T}, \\
M_{5 i} & =\operatorname{diag}\left\{P_{1}^{-1}, \ldots, P_{s}^{-1}\right\} .
\end{aligned}
$$

Performing matrix elementary transformation to the above inequality, we have

$$
\left[\begin{array}{ccccc}
-(1+\alpha) P_{i}+Q & M_{1 i}^{T} & 0 & 0 & 0 \\
M_{1 i} & -M_{5 i} & M_{3 i} & M_{4 i} & M_{2 i} \\
0 & M_{3 i}^{T} & -(1+\alpha) S & 0 & 0 \\
0 & M_{4 i}^{T} & 0 & -(1+\alpha) R & 0 \\
0 & M_{2 i}^{T} & 0 & 0 & -Q
\end{array}\right] \leq 0
$$

Performing a congruence to the above condition by $\operatorname{diag}\left\{P_{i}^{-1} \quad I \quad I \quad I \quad I\right\}$, using Schur complement, and letting $X_{i}=P_{i}^{-1}$ and $Y_{i}=K_{i} X_{i}$, we get

$$
\left[\begin{array}{cccc}
-(1+\alpha) X_{i}+X_{i} Q X_{i} & N_{1 i}^{T} & 0 & 0 \\
N_{1 i} & -M_{5 i}+N_{5 i} & M_{3 i} & M_{4 i} \\
0 & M_{3 i}^{T} & -(1+\alpha) S & 0 \\
0 & M_{4 i}^{T} & 0 & -(1+\alpha) R
\end{array}\right]
$$

$\leq 0$, 
where

$$
\begin{gathered}
N_{1 i}=\left[\sqrt{\pi_{i 1}}\left(\widetilde{A}_{i} X_{i}+B_{i} Y_{i}\right)^{T}, \ldots, \sqrt{\pi_{i s}}\left(\widetilde{A}_{i} X_{i}+B_{i} Y_{i}\right)^{T}\right]^{T}, \\
N_{5 i} \\
=\left[\begin{array}{cccc}
\pi_{i 1} A_{d i} H A_{d i}^{T} & \sqrt{\pi_{i 1}} \sqrt{\pi_{i 2}} A_{d i} H A_{d i}^{T} & \cdots & \sqrt{\pi_{i 1}} \sqrt{\pi_{i s}} A_{d i} H A_{d i}^{T} \\
\sqrt{\pi_{i 2}} \sqrt{\pi_{i 1}} A_{d i} H A_{d i}^{T} & \pi_{i 2} A_{d i} H A_{d i}^{T} & \cdots & \sqrt{\pi_{i 2}} \sqrt{\pi_{i s}} A_{d i} H A_{d i}^{T} \\
\vdots & \vdots & \ddots & \vdots \\
\sqrt{\pi_{i s}} \sqrt{\pi_{i 1}} A_{d i} H A_{d i}^{T} & \sqrt{\pi_{i s}} \sqrt{\pi_{i 2}} A_{d i} H A_{d i}^{T} & & \pi_{i s} A_{d i} H A_{d i}^{T}
\end{array}\right], \\
\widetilde{A}_{i}=\sum_{\sigma_{i} \in \Theta} \mu_{\sigma_{i}} A_{\sigma_{i}}+A_{i} .
\end{gathered}
$$

By using Schur complement to (36) and letting $H=Q^{-1}$, we obtain the linear matrix inequality (30) in Theorem 5.

On the other hand, we consider

$$
\begin{aligned}
& \lambda_{\max }\left(\widetilde{X}_{i}\right)=\frac{1}{\lambda_{\min }\left(\widetilde{P}_{i}\right)}, \\
& \widetilde{X}_{i}=\widetilde{P}_{i}^{-1}=G^{1 / 2} X_{i} G^{1 / 2} .
\end{aligned}
$$

Condition (22) follows that

$$
\begin{gathered}
\frac{c_{1}^{2}}{\min _{i \in M}\left\{\lambda_{\min }\left(\widetilde{X}_{i}\right)\right\}}+c_{1}^{2} d \lambda_{\max }(\widetilde{Q})+\delta^{2} \lambda_{\max }(\mathrm{S}) \\
<\frac{c_{2}^{2}}{\max _{i \in M}\left\{\lambda_{\max }\left(\widetilde{X}_{i}\right)\right\}(1+\alpha)^{N}} .
\end{gathered}
$$

It is easy to check that the above inequality is guaranteed by imposing the following conditions

$$
\begin{array}{cc}
\lambda_{\max }\left(\widetilde{X}_{i}\right)<1, & \lambda_{1}<\lambda_{\min }\left(\widetilde{X}_{i}\right), \\
\lambda_{\max }(\widetilde{Q})<\lambda_{2}, & \lambda_{\max }(S)<\lambda_{3}, \\
\frac{c_{1}^{2}}{\lambda_{1}}+c_{1}^{2} d \lambda_{2}+\delta^{2} \lambda_{3}<\frac{c_{2}^{2}}{(1+\alpha)^{N}}
\end{array}
$$

which are equivalent to conditions (31)-(32). This completes the proof.

Remark 6. It is worth pointing out that Theorem 5 is not a delay-dependent sufficient criterion, which is conservative when the delay is small. Delay-dependent result can be developed in the same way by choosing a Lyapunov functional that includes more entries, as was done in [36], or delay fractioning approach that can be employed as was done in [31-33].

Remark 7. The coupling relationship between time delay and given finite-time horizon of the underlying system is obtained through a finite-time stable constraint (32) in Theorem 5. From condition (32), it can be seen that, in given finite-time horizon, if the time delay $d$ is larger, constraint (32) is more difficult to be satisfied, which means that the existence of time delay increases the instability of system.

\section{Numerical Example}

Consider discrete-time Markov jump nonlinear system (1) with three operation modes and the following data:

$$
\begin{aligned}
& A_{1}=\left[\begin{array}{ll}
0.88 & -0.05 \\
0.40 & -0.72
\end{array}\right], \quad A_{d 1}=\left[\begin{array}{cc}
-0.2 & 0.1 \\
0.2 & 0.15
\end{array}\right], \\
& B_{1}=\left[\begin{array}{l}
2 \\
1
\end{array}\right], \quad B_{w 1}=\left[\begin{array}{l}
0.4 \\
0.5
\end{array}\right], \quad C_{1}=\left[\begin{array}{c}
0 \\
0.1
\end{array}\right] \text {, } \\
& A_{2}=\left[\begin{array}{cc}
2 & 0.24 \\
0.80 & 0.32
\end{array}\right], \quad A_{d 2}=\left[\begin{array}{cc}
-0.6 & 0.4 \\
0.2 & 0.6
\end{array}\right] \text {, } \\
& B_{2}=\left[\begin{array}{c}
1 \\
-1
\end{array}\right], \quad B_{w 2}=\left[\begin{array}{l}
0.2 \\
0.6
\end{array}\right], \quad C_{2}=\left[\begin{array}{c}
0 \\
0.3
\end{array}\right] \text {, } \\
& A_{3}=\left[\begin{array}{ll}
-0.8 & 0.16 \\
0.80 & 0.64
\end{array}\right], \quad A_{d 3}=\left[\begin{array}{cc}
-0.3 & 0.1 \\
0.2 & 0.5
\end{array}\right] \text {, } \\
& B_{3}=\left[\begin{array}{l}
1 \\
1
\end{array}\right], \quad B_{w 2}=\left[\begin{array}{l}
0.1 \\
0.3
\end{array}\right], \quad C_{3}=\left[\begin{array}{c}
0 \\
0.5
\end{array}\right] \text {, } \\
& f_{1}\left(x_{k}\right)=f_{2}\left(x_{k}\right)=f_{3}\left(x_{k}\right)=\sin \left(x_{1 k}\right) \cos \left(x_{2 k}\right) \text {. }
\end{aligned}
$$

Now, a single hidden layer neural network with 2 hidden neurons was chosen to approximate the nonlinear functions $f_{i}\left(x_{k}\right)$. All parameters of activation functions associated with the hidden layer were chosen to be $q_{i l}=0.5$ and $\lambda_{i l}=1$. For these activation functions, we have $s_{i l}\left(0, \phi_{i l}\right)=0$ and $s_{i l}\left(1, \phi_{i l}\right)=1$. The connection weights are trained offline by using BP algorithm. The initial weights and state vector are placed by uniformly distributed random numbers in $\left[\begin{array}{ll}-1 & 1\end{array}\right]$. After 1000 training steps, the optimal approximation weights are as follows:

$$
\begin{aligned}
& W_{1}^{*}=\left[\begin{array}{cc}
-0.86017 & -0.81881 \\
-0.95025 & 0.96405
\end{array}\right], \\
& W_{2}^{*}=\left[\begin{array}{ll}
-0.57752 & -0.58342
\end{array}\right] .
\end{aligned}
$$

The upper bound of approximation error is estimated as $\rho_{i}=$ 0.022 . Obviously, in this case, we have $\Theta=2^{2} \times 2^{1}$. According to (15), $A_{\sigma_{i}}$ can be obtained as follows:

$$
\begin{array}{r}
A_{i 1}=A_{i 2}=A_{i 3}=A_{i 4}=A_{i 5}=A_{1 \oplus[0,0,0]^{T}} \\
=A_{0 \oplus[i, j, k]^{T}}\left[\begin{array}{ll}
0 & 0 \\
0 & 0
\end{array}\right], \quad(i, j, k \in\{0,1\}), \\
A_{i 6}=A_{1 \oplus[1,0]^{T}}=\left[\begin{array}{cc}
0 & 0 \\
0.49677 & 0.47288
\end{array}\right], \\
A_{i 7}=A_{1 \oplus[0,1]^{T}}=\left[\begin{array}{cc}
0 & 0 \\
0.55439 & -0.56245
\end{array}\right], \\
A_{i 8}=A_{1 \oplus[1,1]^{T}}=\left[\begin{array}{cc}
0 & 0 \\
1.0512 & -0.089567
\end{array}\right] .
\end{array}
$$

The initial state and initial mode are taken as $x_{0}=$ $\left[\begin{array}{ll}-0.3 & 0.4\end{array}\right]^{T}$ and $r_{0}=1$, respectively. The iterative step is taken as $N=7$. The mode path from time step 0 to time 


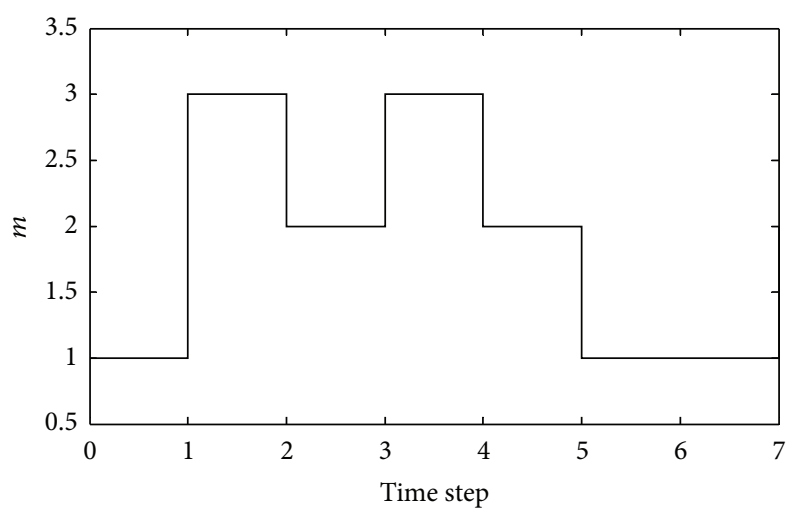

FIGURE 1: Jump modes.

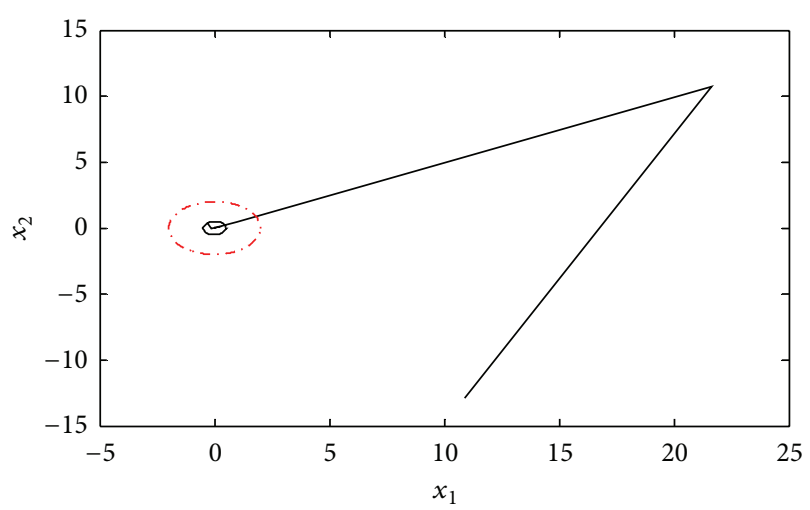

FIgURE 2: State trajectory of free MJS.

step 7 is generated randomly and it is shown in Figure 1. Let $c_{1}=0.5, c_{2}=2, N=7, G=\mathrm{I}, d=0.5, \alpha=0.5$, and $\delta^{2}=1$. By solving the matrix inequalities in Theorem 5 , we have the following controller gains:

$$
\begin{aligned}
K_{1} & =\left[\begin{array}{ll}
-0.9304 & -0.0683
\end{array}\right], \\
K_{2} & =\left[\begin{array}{ll}
-1.7231 & 0.3654
\end{array}\right], \\
K_{3} & =\left[\begin{array}{ll}
1.1486 & -0.1588
\end{array}\right] .
\end{aligned}
$$

The state trajectories of the free and controlled MJLS (16) are drawn in Figures 2 and 3, respectively. It could be seen that the free MJLS (16) is not stochastic FTB because the trajectory exceeds the given bound $c_{2}^{2}$. However, the trajectory is limited between the two ellipsoids regions by employing the proposed control move which satisfactorily justify that the closed-loop MJLS (16) is stochastic FTB.

It should be pointed out that, in the simulation example, as long as the choice of initial condition is satisfied with $\left\|x_{0}^{T} R_{r} x_{0}\right\| \leq c_{1}$, then the system is robustly finite-time stabilizable; that is, system trajectories stay within a given bound.

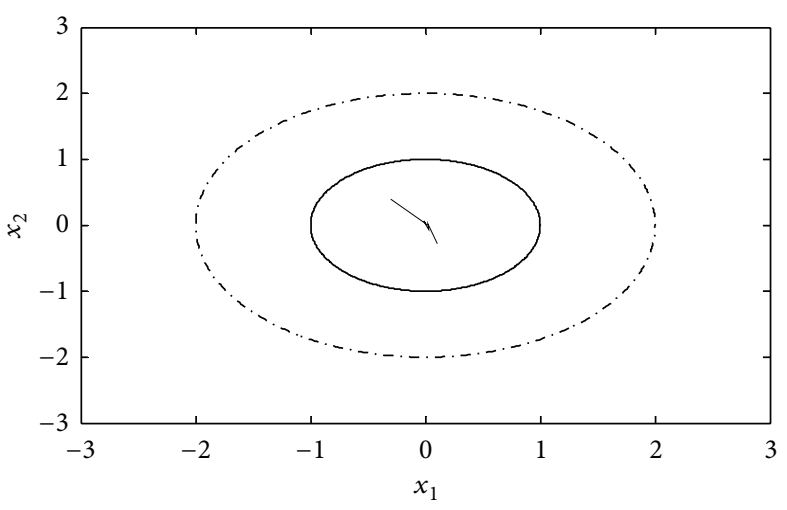

FIGURE 3: State trajectory under finite-time control.

\section{Conclusions}

The finite-time stabilization problem for discrete-time Markovian jump nonlinear system with time delay and normbounded exogenous disturbance is investigated in this paper. The nonlinearities are parameterized by multilayer neural network and the relationship between time delay and given finite-time horizon is explored with delay-independent conditions. The proposed framework is versatile and can accommodate a number of challenging design problems including finite-time control and filtering of discrete-time or continuous-time nonlinear MJS with parameter uncertainties, time delays, and so on. The future work can consider some delay-dependent approaches or delay fractioning approaches to reduce the conservativeness introduced by time delay.

\section{Acknowledgments}

This work was partially supported by the National Natural Science Foundation of China (Grant no. 61104121) and the 111 Project (Grant no. B12018), and the third author would also like to thank the Alexander-von-Humboldt Foundation for providing the support to this research.

\section{References}

[1] W. P. Blair Jr. and D. D. Sworder, "Feedback control of a class of linear discrete systems with jump parameters and quadratic cost criteria," International Journal of Control, vol. 21, pp. 833841, 1975.

[2] D. D. Sworder and R. O. Rogers, "An LQG solution to a control problem with solar thermal receiver," IEEE Transactions on Automatic Control, vol. 28, no. 10, pp. 971-978, 1983.

[3] M. Athans, "Command and control theory: a challenge to control science," IEEE Transactions on Automatic Control, vol. 32, no. 4, pp. 286-293, 1987.

[4] W. Assawinchaichote, S. K. Nguang, and P. Shi, "Robust $H_{\infty}$ fuzzy filter design for uncertain nonlinear singularly perturbed systems with Markovian jumps: an LMI approach," Information Sciences, vol. 177, no. 7, pp. 1699-1714, 2007.

[5] N. M. Krasovskii and E. A. Lidskii, "Analytical design of controllers in systems with random attributes," Automation and 
Remote Control, vol. 22, no. 1-3, pp. 1021-1025, 1141-1146, 12891294, 1961.

[6] Y. Ji and H. J. Chizeck, "Controllability, stabilizability, and continuous-time Markovian jump linear quadratic control," IEEE Transactions on Automatic Control, vol. 35, no. 7, pp. 777788,1990

[7] P. Shi, Y. Xia, G. P. Liu, and D. Rees, "On designing of slidingmode control for stochastic jump systems," IEEE Transactions on Automatic Control, vol. 51, no. 1, pp. 97-103, 2006.

[8] M. S. Mahmoud, P. Shi, E. K. Boukas, and L. Jain, "Simultaneous $\mathrm{H}_{2} / \mathrm{H}_{\infty}$ control of uncertain jump systems with functional time-delays," International Journal of Robust and Nonlinear Control, vol. 18, no. 3, pp. 296-318, 2008.

[9] X. Luan, F. Liu, and P. Shi, "Neural network based stochastic optimal control for nonlinear Markov jump systems," International Journal of Innovative Computing, Information and Control, vol. 6, no. 8, pp. 3715-3723, 2010.

[10] J. Liu, Z. Gu, and S. Hu, " $H_{\infty}$ filtering for Markovian jump systems with time-varying delays," International Journal of Innovative Computing, Information and Control, vol. 7, no. 3, pp. 1299-1310, 2011.

[11] Y. Hu, J. Lam, and J. Liang, "Consensus control of multi-agent systems with missing data in actuators and Markovian communication failure," International Journal of Systems Science, vol. 44, no. 10, pp. 1867-1878, 2013.

[12] X. He, Z. Wang, and D. H. Zhou, "Robust fault detection for networked systems with communication delay and data missing," Automatica, vol. 45, no. 11, pp. 2634-2639, 2009.

[13] L. Zhang and E.-K. Boukas, "Stability and stabilization of Markovian jump linear systems with partly unknown transition probabilities," Automatica, vol. 45, no. 2, pp. 463-468, 2009.

[14] M. Q. Shen and G. H. Yang, "Nonfragile $H_{\infty}$ filtering of continuous Markov jump linear systems with general transition probabilities, Journal of Dynamic Systems," Measurement, and Control, vol. 135, no. 3, Article ID 031005, 2013.

[15] M. Shen and D. Ye, "Improved fuzzy control design for nonlinear Markovian-jump systems with incomplete transition descriptions," Fuzzy Sets and Systems, vol. 217, pp. 80-95, 2013.

[16] L. X. Zhang and J. Lam, "Necessary and sufficient conditions for analysis and synthesis of Markov jump linear systems with incomplete transition descriptions," IEEE Transactions on Automatic Control, vol. 55, no. 7, pp. 1695-1701, 2010.

[17] L. X. Zhang, E.-K. Boukas, and J. Lam, "Analysis and synthesis of Markov jump linear systems with time-varying delays and partially known transition probabilities," IEEE Transactions on Automatic Control, vol. 53, no. 10, pp. 2458-2464, 2008.

[18] L. X. Zhang and E.-K. Boukas, "Mode-dependent $H_{\infty}$ filtering for discrete-time Markovian jump linear systems with partly unknown transition probabilities," Automatica, vol. 45, no. 6 , pp. 1462-1467, 2009.

[19] P. Dorato, "Short time stability in linear time-varying systems," in Proceedings of the of the IRE international Convention Record, Part 4, pp. 83-87, New York, NY, USA, 1961.

[20] F. Amato, M. Ariola, and P. Dorato, "Finite-time control of linear systems subject to parametric uncertainties and disturbances," Automatica, vol. 37, no. 9, pp. 1459-1463, 2001.

[21] F. Amato and M. Ariola, "Finite-time control of discrete-time linear systems," IEEE Transactions on Automatic Control, vol. 50, no. 5, pp. 724-729, 2005.

[22] E. Moulay, M. Dambrine, N. Yeganefar, and W. Perruquetti, "Finite-time stability and stabilization of time-delay systems," Systems \& Control Letters, vol. 57, no. 7, pp. 561-566, 2008.
[23] X. L. Luan, F. Liu, and P. Shi, "Robust finite-time $H_{\infty}$ control for nonlinear jump systems via neural networks," Circuits, Systems, and Signal Processing, vol. 29, no. 3, pp. 481-498, 2010.

[24] X. F. Zhang, G. Feng, and Y. H. Sun, "Finite-time stabilization by state feedback control for a class of time-varying nonlinear systems," Automatica, vol. 48, no. 3, pp. 499-504, 2012.

[25] F. Amato, R. Ambrosino, M. Ariola, and C. Cosentino, "Finitetime stability of linear time-varying systems with jumps," Automatica, vol. 45, no. 5, pp. 1354-1358, 2009.

[26] L. L. Hou, G. D. Zong, and Y. Q. Wu, "Finite-time control for switched delay systems via dynamic Output feedback," International Journal of Innovative Computing, Information and Control, vol. 8, no. 7, pp. 4901-4913, 2012.

[27] W. H. Zhang and X. Y. An, "Finite-time control of linear stochastic systems," International Journal of Innovative Computing, Information and Control, vol. 4, no. 3, pp. 689-696, 2008.

[28] X.-L. Luan, F. Liu, and P. Shi, "Finite-time stabilization of stochastic systems with partially known transition probabilities," Journal of Dynamic Systems, Measurement and Control, vol. 133, no. 1, Article ID 014504, 2011.

[29] D. Yang and K.-Y. Cai, "Finite-time quantized guaranteed cost fuzzy control for continuous-time nonlinear systems," Expert Systems with Applications, vol. 37, no. 10, pp. 6963-6967, 2010.

[30] X. Luan, F. Liu, and P. Shi, "Neural-network-based finite-time $H_{\infty}$ control for extended Markov jump nonlinear systems," International Journal of Adaptive Control and Signal Processing, vol. 24, no. 7, pp. 554-567, 2010.

[31] J. Hu, Z. Wang, H. Gao, and L. K. Stergioulas, "Robust sliding mode control for discrete stochastic systems with mixed time delays, randomly occurring uncertainties, and randomly occurring nonlinearities," IEEE Transactions on Industrial Electronics, vol. 59, no. 7, pp. 3008-3015, 2012.

[32] J. Hu, Z. D. Wang, and H. J. Gao, "A delay fractioning approach to robust sliding mode control for discrete-time stochastic systems with randomly occurring non-linearities," IMA Journal of Mathematical Control and Information, vol. 28, no. 3, pp. 345363, 2011.

[33] J. Hu, Z. D. Wang, Y. G. Niu, and L. K. Stergioulas, " $H_{\infty}$ sliding mode observer design for a class of nonlinear discrete timedelay systems: a delay-fractioning approach," International Journal of Robust and Nonlinear Control, vol. 22, no. 16, pp. 18061826, 2012.

[34] S. Limanond and J. Si, "Neural-network-based control design: an LMI approach," IEEE Transactions on Neural Networks, vol. 9, no. 6, pp. 1422-1429, 1998.

[35] A. R. Barron, "Approximation and estimation bounds for artificial neural networks," Machine Learning, vol. 14, no. 1, pp. 115-133, 1994.

[36] L. Zhang, E.-K. Boukas, and A. Haidar, "Delay-rangedependent control synthesis for time-delay systems with actuator saturation," Automatica, vol. 44, no. 10, pp. 2691-2695, 2008. 


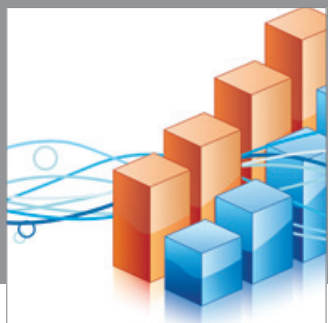

Advances in

Operations Research

mansans

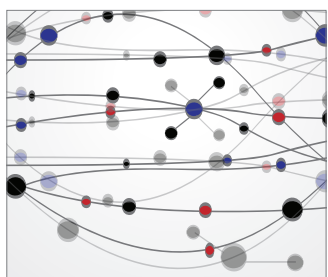

The Scientific World Journal
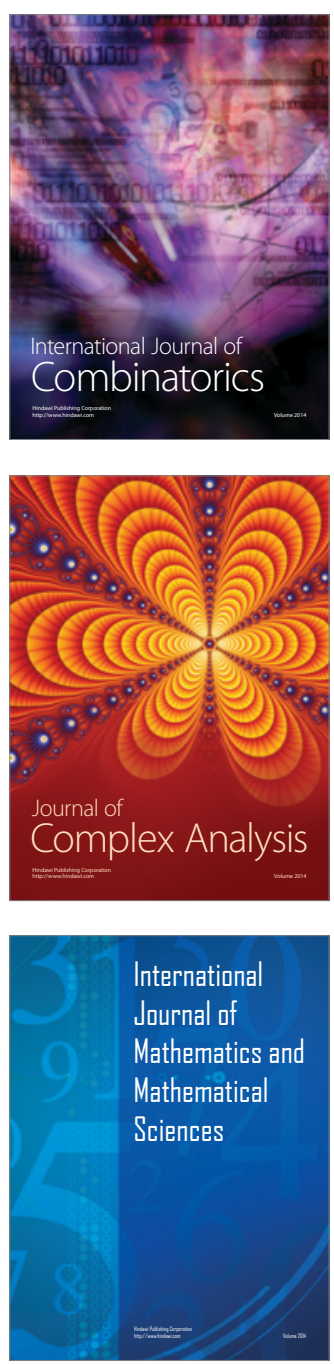
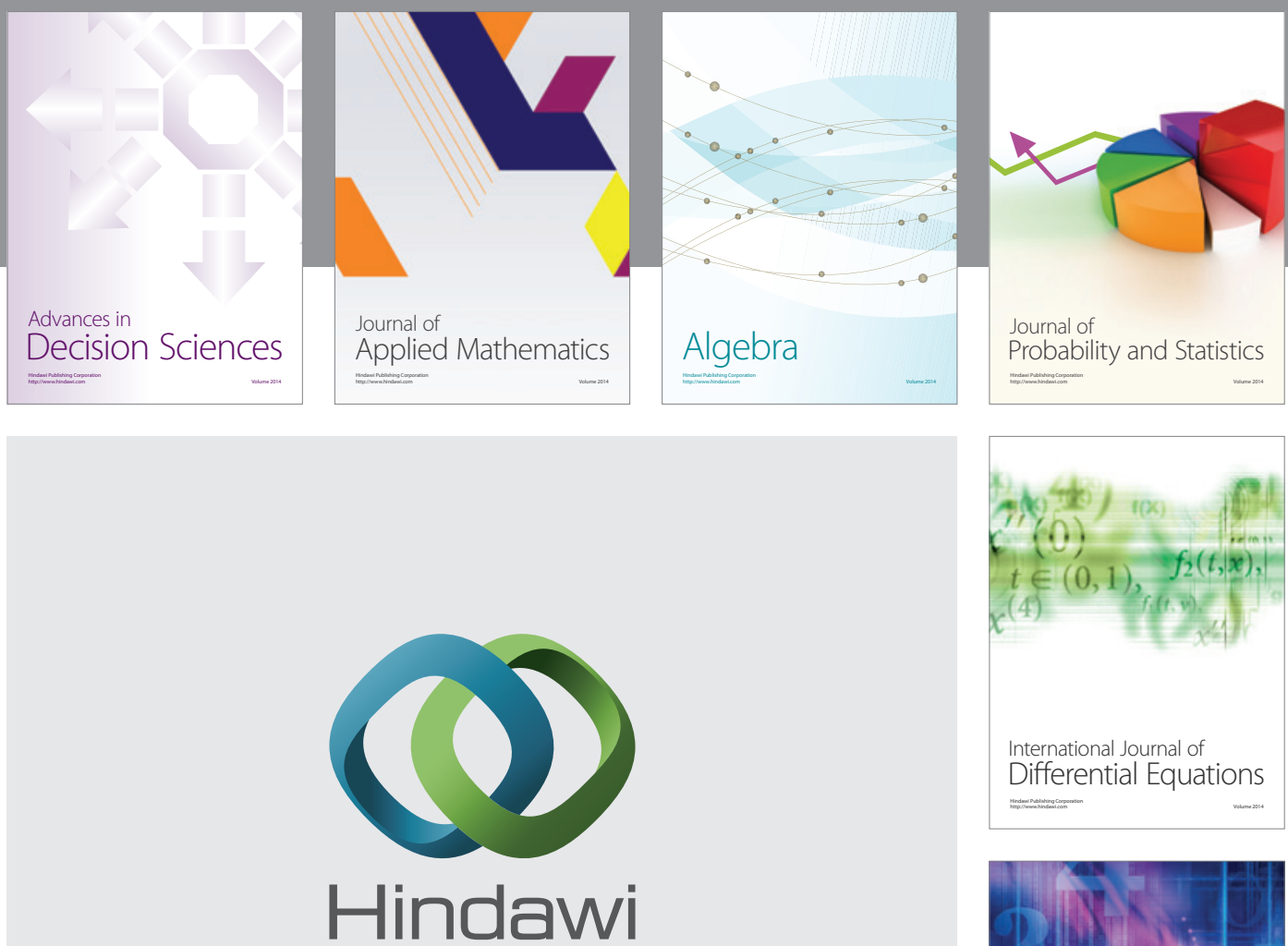

Submit your manuscripts at http://www.hindawi.com
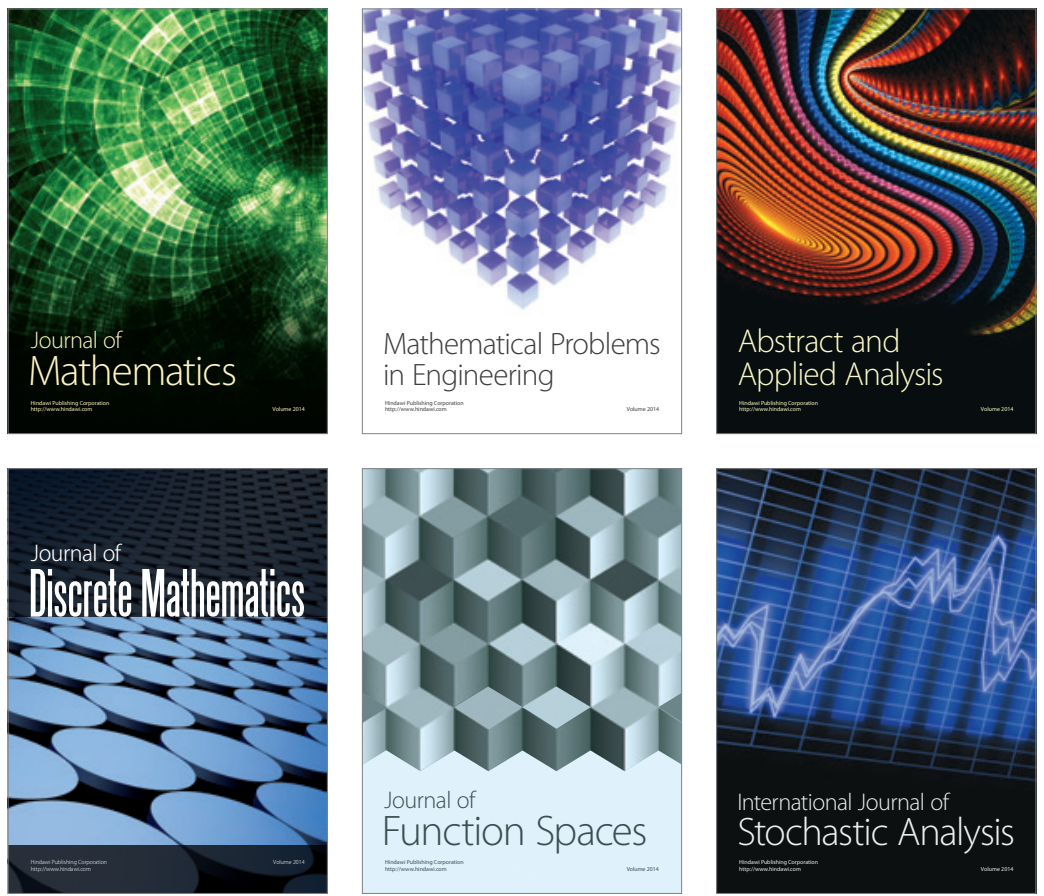

Journal of

Function Spaces

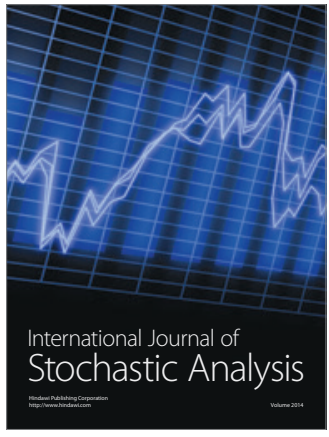

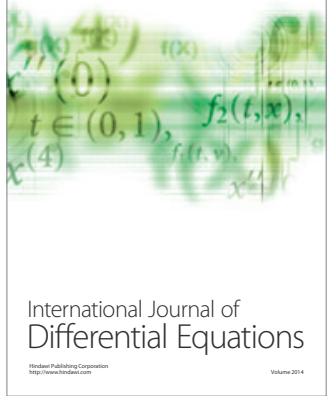
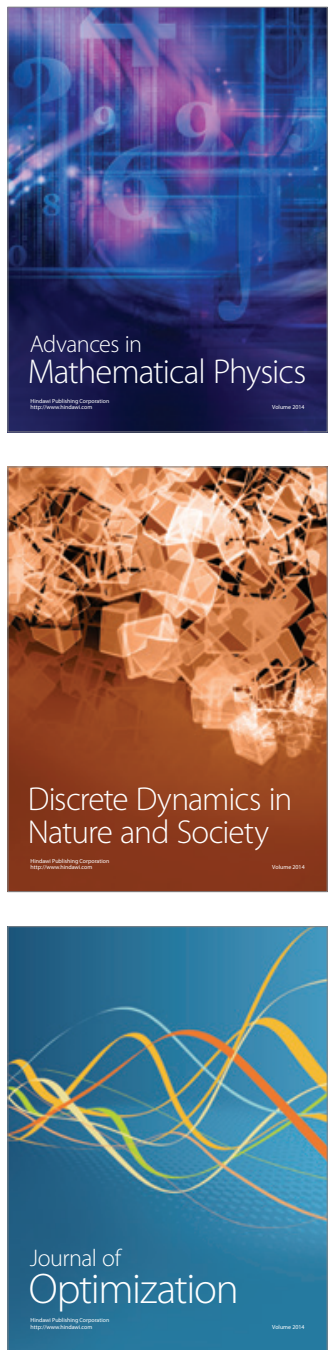